high power fields were scored for fibrosis, inflammation, BiP and GADD34 using semi-quantitative analysis by 2 blinded, independent investigators. Data were analysed by linear regression using Prism software.

$\mathrm{BiP}$ and GADD34 were localised to reactive type II pneumocytes and columnar epithelium within areas of fibrosis. GADD34 was also evident in the endothelium. No staining was detected in fibroblasts. Epithelial GADD34 correlated with extent of fibrosis in familial pulmonary fibrosis $\left(\mathrm{r}^{2}=0.72, \mathrm{p}<0.001\right)$, IPF $\left(\mathrm{r}^{2}=0.51, \mathrm{p}<0.0001\right)$ and NSIP $\left(\mathrm{r}^{2}=0.46, \mathrm{p}<0.0001\right)$. In contrast, BiP was associated with fibrosis in IPF $\left(\mathrm{r}^{2}=0.49\right.$, $\mathrm{p}<0.0001)$ and HP $\left(\mathrm{r}^{2}=0.59, \mathrm{p}<0.0001\right)$.

These data show that ER stress and the unfolded protein response are associated with fibrosis in ILD. Hence targeting ER stress may be a novel therapeutic option for pulmonary fibrosis.

\section{S77 MODULATORY EFFECTS OF RHEUMATOID ARTHRITIS IgG ON NEUTROPHIL ACTIVATION: A POTENTIAL ROLE IN RA-ILD}

\footnotetext{
${ }^{1,2} \mathrm{AA}$ Khawaja, ${ }^{2} \mathrm{C}$ Pericleous, ${ }^{2} \mathrm{VM}$ Ripoll, ${ }^{3} \mathrm{HL}$ Booth, ${ }^{3} \mathrm{~V}$ Holmes, ${ }^{1} \mathrm{~T}$ Mikolasch, ${ }^{2} \mathrm{I}$ Giles, ${ }^{1} \mathrm{JC}$ Porter. ${ }^{1}$ Centre for Inflammation and Tissue Repair, University College London, London, UK; ${ }^{2}$ Centre for Rheumatology, University College London, London, UK; ${ }^{3}$ Department of Thoracic Medicine, University College London Hospital, London, UK
}

\subsection{6/thoraxjnl-2016-209333.83}

Background Rheumatoid arthritis (RA) is an autoimmune rheumatic disease (ARD) characterised by circulating autoantibodies, including anti-citrullinated protein antibodies (ACPA). Many RA patients have extra-articular disease, including $\sim 10 \%$ with interstitial lung disease (ILD). Risk factors for RA-ILD include: male sex; age; smoking history and ACPA positivity.

Hypothesis We propose that RA-IgG, including ACPA, modulate neutrophil functions including: generation of reactive oxygen species (ROS), neutrophil adhesion and generation of neutrophil extracellular traps (NETs) that may contribute to joint and extraarticular tissue damage, including ILD.

Methods Neutrophils and IgG were isolated from RA patients or healthy controls (HC). Bronchoalveolar lavage (BAL) was performed on ILD patients and controls underdoing bronchoscopy for other reasons. ROS production was measured using an enzymatic assay to assess hydrogen peroxide ( $\mathrm{H} 2 \mathrm{O} 2)$ generation. Neutrophil integrins were quantified by flow cytometry. Effects of purified IgG upon neutrophil adhesion to immobilised fibrinogen (Mac-1/aMß2-dependent) and fibronectin (VLA-4/a4ß1dependent) were determined using a fluorescent adhesion assay. NETosis was measured using a novel capture ELISA.

Results We demonstrated increased NETs in the BAL of patients with active ILD ( $\mathrm{n}=3$; ARD-ILD) compared to those with inactive disease ( $n=2$; IPF and ARD-ILD) or controls $(n=1$; CLL). In addition, we showed binding of RA-IgG to control neutrophils, which increased with neutrophil activation. Stimulation of HC $(n=12)$ and RA $(n=7)$ neutrophils with PMA produced similar rates of $\mathrm{H} 2 \mathrm{O} 2$ generation $(\mathrm{p}=0.9939)$. Exposure of $\mathrm{HC}$ neutrophils to RA-IgG $(n=9)$ however, increased $\mathrm{H} 2 \mathrm{O} 2$ production compared to HC-IgG $(\mathrm{n}=9)(\mathrm{p}<0.0001)$, which was not blocked by FcR blockade. RA-IgG also enhanced PMA-stimulated adhesion of HC neutrophils to fibrinogen ( $p=0.0028)$ and fibronectin $(\mathrm{p}=0.0024)$, which was inhibited by aMß2 or $\beta 1$ integrin blockade respectively. RA-IgG increased both spontaneous $(\mathrm{p}=0.0248)$ and PMA-induced $(\mathrm{p}=0.0200)$ NETosis of HC neutrophils compared to HC-IgG. Immunofluorescence studies demonstrate that aMß2 activation induces NETosis. Further examination found that NETosis could be suppressed by $\mathrm{p} 38$ MAPK inhibition $(\mathrm{p}=0.0034)$.

Conclusion We have shown that RA-IgG modulates several aspects of neutrophil activation and function. In addition, we found increased neutrophil activation in the lungs of patients with active ARD-ILD. Further work is underway to evaluate the contribution of these processes to the pathogenesis of RA-ILD.

\section{S78 LYMPHOPAENIA AND INCREASED ACE LEVELS STRATIFY SARCOIDOSIS PATIENTS TO UNDERLYING INCREASE IN IFN- $\gamma+$ LYMPHOCYTE AND TNF- $\alpha+$ MONOCYTES RESPECTIVELY}

${ }^{1}$ YK Kendrick, ${ }^{2} \mathrm{SL}$ Cole, ${ }^{3} \mathrm{R}$ Hoyles, ${ }^{1} \mathrm{LP}$ Ho. ${ }^{1} \mathrm{MRC}$ Human Immunology Unit, Weatherall Institute of Molecular Medicine, University of Oxford and Oxford Interstitial Lung Disease Service, Oxford, UK; ${ }^{2}$ MRC Human Immunology Unit, Weatherall Institute of Molecular Medicine, University of Oxford, Oxford, UK; ${ }^{3}$ Oxford Interstitial Lung Disease Service, OUH NHS FoundationTrust, Oxford, UK

\subsection{6/thoraxjnl-2016-209333.84}

Introduction Sarcoidosis is a heterogeneous disease, and different mechanisms may contribute to disease activity at any one time. Both TH1 lymphocyte (IFN- $\gamma+$, IL-2+ CD4 T cells) and activated tissue macrophages contribute to the formation of granuloma and are established disease pathways. More recently, monocytes (precursors of tissue macrophages) have also been implicated. We reasoned that if we could identify a commonly used clinical test as a biomarker for these disease pathways, it could be used as a disease activity marker, and to guide evaluation of new therapies and repositioning of current drugs. In this study, we question whether serum ACE and circulating lymphocyte count correlated with different cellular immune function.

Methods 44 consecutive patients fulfilling the ATS-WASOG diagnostic criteria for sarcoidosis within a 2-year period were recruited. Patients on treatment, current cigarette smokers, intercurrent immune disease and malignancies were excluded. Blood samples from all patients were processed contemporaneously for ACE, lymphocyte counts and CD4 T cell intra-cellular cytokine staining (ICS) for IFN- $\gamma$, IL-2 and IL-17 and CD14hi monocytes ICS for TNF- $\alpha$.

Results and discussion We found no correlation between lymphocyte count and ACE $(\mathrm{r}=-0.2 ; \mathrm{p}=0.86)$ suggesting that these two abnormalities reflected independent processes. Lymphopaenia was significantly correlated with markers of activated CD4 T cells (IFN- $\gamma$, IL2 + and TNF- $\alpha+)(r=-0.50,0.50$ and 0.60 respectively; all $\mathrm{p}<0.001$; Spearman Rank Sum test); while ACE only correlated with level of TNF- $\alpha+$ monocytes $(r=0.60$; $\mathrm{p}<0.0001)$.

Conclusions These results suggest that high ACE and lymphopaenia (i) reflect disease activity (ii) are likely to be endpoints of two different mechanistic pathways, and (iii) that they could potentially stratify patients into those with lymphocytic dominant and monocyte dominant disease processes. Thus a monocyte pathway inhibitor could be used specifically in patients with high ACE levels while drugs that target T cell activity may be targeted to those with lymphopaenia. 